\title{
真子群的导群都较小的有限 $p$-群
}

\section{张军强, 黎先华*}

苏州大学数学科学学院, 苏州 215006

E-mail: xhli@suda.edu.cn

收稿日期: 2008-12-22；接受日期: 2009-04-13; * 通信作者

国家自然科学基金 (批准号: 10571128 和 10871032), 江苏省自然科学基金 (批准号: BK2008156) 和苏州市高层次人 才资助项目

摘要 设 $G$ 是一个有限 $p$-群. 若 $G$ 的真子群的导群的阶都整除 $p^{i}$, 则称 $G$ 为 $D_{i}$-群. 我 们给出了所有 $D_{1}$-群的一个刻画. 这回答了 Berkovich 提出的一个问题.

关键词 有限 $p$-群 导群 内交换 $p$-群 $D_{1}$-群

$\mathrm{MSC}(2000)$ 主题分类 $20 \mathrm{D} 15,20 \mathrm{D} 10$

\section{1 引言}

本文所考虑的群皆为有限 $p$-群. $G$ 表示一个 $p$-群. $C_{p^{n}}$ 表示 $p^{n}$ 阶循环 $p$-群, $C_{p}^{n}$ 表示 $p^{n}$ 阶初等交换 $p$-群. $d(G)$ 表示 $G$ 的极小生成元的个数, $\Phi(G)$ 表示 $G$ 的 Frattini 子群. 我 们有 $p^{d(G)}=|G / \Phi(G)|$. 其它的术语和符号是标准的 (参见文献 [1]).

群的交换性由它的导群来反映. 比如, 群 $G$ 是交换的当且仅当 $G^{\prime}=1$. 非交换群 $G$ 称 为内交换群, 如果对 $G$ 的任意真子群 $H$ 有 $H^{\prime}=1$.

1947 年, Rédei ${ }^{[2]}$ 给出了内交换 $p$-群的分类.

定理 $1.1^{[2]}$ 设 $G$ 是一个内交换 $p$-群. 那么 $d(G)=2,\left|G^{\prime}\right|=p$ 并且 $G$ 为下列情况之 一:

(i) $G$ 同构于 $Q_{8}$;

(ii) $G=\left\langle a, b \mid a^{p^{n}}=b^{p^{m}}=1,[a, b]=a^{p^{n-1}}\right\rangle, n \geqslant 2, m \geqslant 1,|G|=p^{m+n}$, 并且 $G$ 亚循环;

(iii) $G=\left\langle a, b \mid a^{p^{n}}=b^{p^{m}}=c^{p}=1,[a, b]=c,[c, a]=[c, b]=1\right\rangle, G$ 非亚循环, $|G|=$ $p^{m+n+1}$, 并且如果 $p=2$, 那么有 $m+n \geqslant 3$.

更进一步地, Berkovich ${ }^{[3]}$ 给出了导群的阶为 $p$ 的有限 $p$-群的结构.

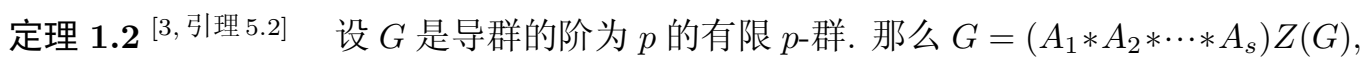
其中 $*$ 表示中心积, $A_{1}, \ldots, A_{s}$ 是 $G$ 的内交换子群. 特别地, $G / Z(G)$ 初等交换. 并且若 $G / G^{\prime}$ 初等交换, 则 $E=A_{1} * A_{2} * \cdots * A_{s}$ 是超特殊 $p$-群.

1999 年, Blackburn 在文献 [4] 中利用导群阶为 $p$ 的有限 $p$-群的同构类集合与满足一定 条件的整向量集合 $\varphi_{n}$ 之间的一一映射也给出了导群阶为 $p$ 的有限 $p$-群结构的刻画. 
接下来, 人们自然想知道真子群的导群的阶都小于或等于 $p$ 的有限 $p$-群的结构. Berkovich 在文献 $[5$, 研究问题与题目 I $]$ 中提出了下面的问题.

问题 39 分类真子群 $H$ 都满足 $\left|H^{\prime}\right| \leqslant p$ 的 $p$-群 $G$.

如果 $p$-群 $G$ 的真子群的导群的阶都整除 $p^{i}$, 则称 $G$ 为 $D_{i}$-群. $D_{1}$-群恰好是上述问题中 要分类的群. 在文献 [6] 中, Berkovich 定义了 $A_{n}$-群的概念. 一个 $p$-群 $G$ 称为 $A_{n}$-群, 如果 $G$ 有一个指数为 $p^{n-1}$ 的非交换子群, 并且所有指数为 $p^{n}$ 的子群都交换. 显然, $A_{1}$-群是内交 换 $p$-群, $A_{2}$-群是 $D_{1}$-群. 本文给出了 $D_{1}$-群的结构. 这回答了问题 39 , 主要结果是定理 3.1 及其推论.

\section{2 预备知识}

引理 2.1 ${ }^{[7]}$ 设 $A$ 是群 $G$ 的一个交换子群, 并且 $G / A=\langle b A\rangle$ 循环, 那么

(i) 映射 $a \mapsto[a, b], a \in A$ 是 $A$ 到 $G^{\prime}$ 的一个满同态;

(ii) $G^{\prime} \cong A /(A \cap Z(G))$ (也可参见文献 [8, 引理 12.12]).

当 $A$ 是 $G$ 的极大子群时, 有下面的结果

引理 2.2 (参见 [5, 引理 1.1]) 设 $G$ 是一个非交换 $p$-群. 若 $G$ 有交换极大子群, 则 $|G|=p \cdot|Z(G)| \cdot\left|G^{\prime}\right|$.

引理 2.3 (参见 [9, 定理 2]) 对非交换 $p$-群 $G$, 下面两个条件等价:

(a) $G$ 亚循环;

(b) 存在 $G^{\prime}$ 中指数为 $p$ 的 $G$ - 不变子群 $R$, 使得 $G / R$ 亚循环.

引理 2.4 (参见 [9, 引理 1 (b)]) 设 $G$ 是一个非交换 $p$-群. 若 $M$ 和 $N$ 是 $G$ 的两个不 同的极大子群, 则 $\left|G^{\prime}\right| \leqslant p\left|M^{\prime} N^{\prime}\right|$.

引理 2.5 (参见 [10, 引理 $3.2(\mathrm{a})]$ ) 设 $G$ 是一个非交换 $p$-群. 若 $G^{\prime} \leqslant Z(G), \exp \left(G^{\prime}\right)=p$ 且 $d(G)=2$, 则 $G$ 为 $A_{1}$-群.

因为 $A_{1}$-群是内交换 $p$-群, 由引理 2.5 和定理 1.1, 我们得到: 一个 $p$-群 $G$ 是内交换的当 且仅当 $d(G)=2$ 且 $\left|G^{\prime}\right|=p$.

引理 2.6 假设 $G$ 是一个 $D_{1}$-群.

(i) $G$ 的任意极大子群 $M$, 有 $\left|M^{\prime}\right|=1$ 或 $p$, 且 $M^{\prime} \leqslant G^{\prime} \cap Z(G)$;

(ii) 若 $H \leqslant G$, 则 $H$ 是 $D_{1}$-群;

(iii) 若 $N \unlhd G$, 则 $G / N$ 也是 $D_{1}$-群.

证明 (i) 由于 $M \triangleleft G$ 且 $M^{\prime}$ 是 $M$ 的一个特征子群, $M^{\prime} \triangleleft G$. 由 $D_{1}$-群的定义, $\left|M^{\prime}\right|=1$ 或 $p$. 于是 $M^{\prime} \leqslant G^{\prime} \cap Z(G)$.

(ii) 和 (iii) 的证明由 $D_{1}$-群的定义即得.

我们将不加声明地使用上述引理.

引理 2.7 假设 $G$ 是一个 $D_{1}$-群. 则

(i) $\left|G^{\prime}\right| \leqslant p^{3}$;

(ii) 若 $\left|G^{\prime}\right| \geqslant p^{2}$, 则 $d(G) \leqslant 3$.

证明 (i) 若 $G$ 的极大子群唯一, 则 $G$ 循环, 结果成立. 假设 $G$ 有两个极大子群 $A$ 和 $B$. 由 $G$ 是一个 $D_{1}$-群, 我们有 $\left|A^{\prime}\right| \leqslant p$ 和 $\left|B^{\prime}\right| \leqslant p$, 因此 $\left|A^{\prime} B^{\prime}\right| \leqslant p^{2}$. 由引理 2.4, 我们得到 $\left|G^{\prime}\right| \leqslant p^{3}$. 
(ii) 假设 $\left|G^{\prime}\right| \geqslant p^{2}$. 由定理 $1.1, G$ 不是内交换 $p$-群. 又因为 $G$ 是 $D_{1}$-群, 所以 $G$ 中存 在极大子群 $A$ 满足 $\left|A^{\prime}\right|=p$. 如果对 $G$ 的任意极大子群 $B$ 都有 $B^{\prime} \leqslant A^{\prime}$, 则 $G / A^{\prime}$ 内交换 或交换. 若 $G / A^{\prime}$ 交换, 则 $G^{\prime} \leqslant A^{\prime}$, 而 $\left|G^{\prime}\right| \geqslant p^{2}$, 这不可能. 因此, $G / A^{\prime}$ 是一个内交换 $p$-群. 由定理 1.1 得 $d\left(G / A^{\prime}\right)=2$. 而 $A^{\prime} \leqslant \Phi(G)$, 所以 $d(G)=d\left(G / A^{\prime}\right)=2$, 结论成立. 假设 $G$ 有 极大子群 $B$ 满足 $B^{\prime} \not \leq A^{\prime}$. 因为 $G$ 是 $D_{1}$-群, 我们有 $\left|A^{\prime}\right|=\left|B^{\prime}\right|=p$ 且 $A^{\prime} \cap B^{\prime}=1$. 选取 $a_{1}, a_{2} \in A$ 和 $a_{3}, a_{4} \in B$ 满足 $\left[a_{1}, a_{2}\right] \neq 1$ 和 $\left[a_{3}, a_{4}\right] \neq 1$, 则 $A^{\prime}=\left\langle\left[a_{1}, a_{2}\right]\right\rangle, B^{\prime}=\left\langle\left[a_{3}, a_{4}\right]\right\rangle$ 且 $\left|\left\langle a_{1}, a_{2}, a_{3}, a_{4}\right\rangle^{\prime}\right| \geqslant p^{2}$. 从而 $G=\left\langle a_{1}, a_{2}, a_{3}, a_{4}\right\rangle, d(G) \leqslant 4$. 接下来我们只需要证明 $d(G) \neq 4$.

假设 $d(G)=4$, 那么有 $\left\langle a_{1}, a_{2}, a_{k}\right\rangle<G,\left\langle a_{3}, a_{4}, a_{s}\right\rangle<G$, 其中 $k \in\{3,4\}, s \in\{1,2\}$. 由 $D_{1}$-群的定义, $\left\langle a_{1}, a_{2}, a_{k}\right\rangle^{\prime}=\left\langle\left[a_{1}, a_{2}\right]\right\rangle,\left\langle a_{3}, a_{4}, a_{s}\right\rangle^{\prime}=\left\langle\left[a_{3}, a_{4}\right]\right\rangle$. 因此 $\left\{\left[a_{1}, a_{k}\right],\left[a_{2}, a_{k}\right]\right\} \subseteq$ $\left\langle\left[a_{1}, a_{2}\right]\right\rangle,\left\{\left[a_{s}, a_{3}\right],\left[a_{s}, a_{4}\right]\right\} \subseteq\left\langle\left[a_{3}, a_{4}\right]\right\rangle$. 所以 $\left[a_{s}, a_{k}\right] \in\left\langle\left[a_{1}, a_{2}\right]\right\rangle \cap\left\langle\left[a_{3}, a_{4}\right]\right\rangle$, 从而 $\left[a_{s}, a_{k}\right]=1$, 其中 $s=1,2 ; k=3,4$. 然而, $\left\langle a_{1}, a_{2} a_{3}, a_{4}\right\rangle$ 是 $G$ 的真子群且 $\left|\left\langle a_{1}, a_{2} a_{3}, a_{4}\right\rangle^{\prime}\right|=p^{2}$, 矛盾. 因此 $d(G) \leqslant 3$. 证明完成.

众所周知, $K_{n}(G)=\left\langle\left[g_{1}, g_{2}, \ldots, g_{n}\right] \mid g_{i} \in G\right\rangle$ 是 $G$ 的下中心群列的第 $n$ 项, $K_{n}(G)=$ $\left[K_{n-1}(G), G\right]$ 且 $G^{\prime}=K_{2}(G)$ 是 $G$ 的导群. 显然, 若 $K_{n+1}(G)=1$, 则 $K_{n}(G) \leqslant Z(G)$.

引理 2.8 假设 $G$ 是一个非交换 $p$-群. 若 $d(G)=2$, 则对任意 $H<G$, 有 $H^{\prime}<G^{\prime}$.

证明 设 $G=\langle a, b\rangle$. 由文献 $\left[1,258\right.$ 页, 辅理 1.11], 我们有 $G^{\prime}=\left\langle[a, b], K_{3}(G)\right\rangle$ 且 $G^{\prime} / K_{3}(G)$ 循环.

若 $K_{3}(G)=1$, 则 $G^{\prime}=\langle[a, b]\rangle$ 循环且 $G^{\prime} \leqslant Z(G)$. 因为 $G / \Phi(G)=\langle\bar{a}\rangle \times\langle\bar{b}\rangle$ 是一个 $p^{2}$ 阶 初等交换 $p$-群, $G$ 的极大子群可以写为 $M=\langle\Phi(G), c\rangle=\Phi(G)\langle c\rangle=G^{\prime} \mho(G)\langle c\rangle$, 其中 $c=a^{i} b^{j}$, $0 \leqslant i, j \leqslant p-1$ 并且 $i \neq 0$ 或 $j \neq 0$. 对任意 $u, v \in M$, 设 $u=x_{1}\left(\Pi_{i=1}^{t} a_{i}^{p}\right) z_{1}, v=x_{2}\left(\Pi_{j=1}^{s} b_{j}^{p}\right) z_{2}$, 其中 $x_{i} \in G^{\prime}, a_{i}, b_{j} \in G, z_{i} \in\langle c\rangle$. 由于 $x_{i} \in Z(G)$, 我们有 $\left[x_{1}\left(\Pi_{i=1}^{t} a_{i}^{p}\right) z_{1}, x_{2}\left(\Pi_{j=1}^{s} b_{j}^{p}\right) z_{2}\right]=$ $\left[\left(\Pi_{i=1}^{t} a_{i}^{p}\right) z_{1},\left(\Pi_{j=1}^{s} b_{j}^{p}\right) z_{2}\right]$. 由 $K_{3}(G)=1, G^{\prime} \leqslant Z(G)$ 和 [1,253 页, 辅理 1.2 和 1.3],

$$
\begin{aligned}
& {[u, v]=\left[\left(\Pi_{i=1}^{t} a_{i}^{p}\right),\left(\Pi_{j=1}^{s} b_{j}^{p}\right) z_{2}\right]\left[z_{1},\left(\Pi_{j=1}^{s} b_{j}^{p}\right) z_{2}\right]} \\
& =\left[\Pi_{i=1}^{t} a_{i}^{p}, \Pi_{j=1}^{s} b_{j}^{p}\right]\left[\Pi_{i=1}^{t} a_{i}^{p}, z_{2}\right]\left[z_{1}, \Pi_{j=1}^{s} b_{j}^{p}\right] \\
& =\Pi_{i, j}\left[a_{i}, b_{j}\right]^{p^{2}} \Pi_{i=1}^{t}\left[a_{i}, z_{2}\right]^{p} \Pi_{j=1}^{s}\left[z_{1}, b_{j}\right]^{p},
\end{aligned}
$$

所以 $[u, v] \in \mho\left(G^{\prime}\right)$. 由 $\mho\left(G^{\prime}\right)<G^{\prime}$, 我们有 $M^{\prime}<G^{\prime}$.

若 $K_{3}(G) \neq 1$, 令 $\bar{G}=G / K_{3}(G)$, 则 $\bar{G}$ 是一个非交换 $p$-群且 $d(\bar{G})=2$. 对任意 $H<G$, 当 $K_{3}(G) \not H^{\prime}$, 我们有 $H^{\prime}<G^{\prime}$; 当 $K_{3}(G)<H^{\prime}$, 对 $|G|$ 作归纳得, $\overline{H^{\prime}}<\overline{G^{\prime}}$, 所以 $H^{\prime}<G^{\prime}$. 结论成立.

注 (1) 应用引理 2.5 , Berkovich 给出引理 2.8 的另一证明. 设 $R<G^{\prime}$ 是 $G^{\prime}$ 中指数为 $p$ 的 $G$ - 不变子群. 那么 $(G / R)^{\prime}$ 的阶为 $p$ 且 $d(G / R)=2$. 由引理 $2.5, G / R$ 内交换. 设 $H$ 是 $G$ 的任意极大子群. 那么 $H^{\prime} \leqslant R<G^{\prime}$.

(2) 由引理 2.8, 我们也可以得到: 若 $G$ 是一个 $p$-群, $d(G)=2$ 且 $\left|G^{\prime}\right|=p$, 则 $G$ 内交换.

引理 2.9 假设 $G$ 是一个有限 $p$-群. 若下列情况之一成立

(i) $\left|G^{\prime}\right| \leqslant p$;

(ii) $d(G)=2,\left|G^{\prime}\right|=p^{2}$;

(iii) $d(G)=2, c(G)=3, G^{\prime} \cong C_{p}^{3}$, 其中 $p>2$;

(iv) $d(G)=3, c(G)=2, G^{\prime} \cong C_{p}^{3}$ 或 $C_{p}^{2}$, 
那么 $G$ 是一个 $D_{1}$-群.

证明 (i) 结论显然.

(ii) 由引理 2.8 即得.

(iii) 设 $G=\langle a, b\rangle$. 因为 $c(G)=3$, 所以 $K_{4}(G)=1, K_{3}(G) \leqslant Z(G)$, 从而对任意 $x \in K_{3}(G)$ 和 $u \in G$, 我们有 $x^{u}=x$. 注意到 $[a, a]=[b, b]=1$. 于是由文献 $[1,258$ 页, 辅 理 1.11], 我们有 $K_{3}(G)=\langle[a, b, b],[a, b, a],[b, a, b],[b, a, a]\rangle$, 且 $G^{\prime}=\left\langle[a, b], K_{3}(G)\right\rangle$. 因为对任 意 $x, y \in G$, 有 $\left[x^{-1}, y\right]=[y, x]^{x^{-1}}=\left([x, y]^{-1}\right)^{x^{-1}}$, 并且 $K_{3}(G) \leqslant Z(G)$, 所以

$$
\begin{aligned}
{[b, a, a] } & =\left[[a, b]^{-1}, a\right]=\left([[a, b], a]^{-1}\right)^{[a, b]^{-1}}=[[a, b], a]^{-1}=[a, b, a]^{-1}, \\
{[b, a, b] } & =\left[[a, b]^{-1}, b\right]=\left([[a, b], b]^{-1}\right)^{[a, b]^{-1}}=[[a, b], b]^{-1}=[a, b, b]^{-1} .
\end{aligned}
$$

于是我们得到 $K_{3}(G)=\langle[a, b, b],[a, b, a]\rangle$, 且 $G^{\prime}=\langle[a, b],[a, b, b],[a, b, a]\rangle$. 由 $K_{3}(G) \leqslant Z(G)$, 容易得到 $G^{\prime}$ 交换. 因此, 对任意的 $x \in G^{\prime}$, 我们可以假设 $x=[a, b]^{i}[a, b, b]^{j}[a, b, a]^{k}$, 其中 $i, j$ 和 $k$ 为整数. 因为 $K_{3}(G) \leqslant Z(G)$, 所以对任意 $g \in G$, 我们有

$$
[x, g]=\left[[a, b]^{i}[a, b, b]^{j}[a, b, a]^{k}, g\right]=\left[[a, b]^{i}, g\right]=[[a, b], g]^{i}=[a, b, g]^{i} \in\langle[a, b, g]\rangle .
$$

这说明 $\left[G^{\prime}, g\right]=\langle[a, b, g]\rangle$. 对任意的 $z, g \in G$ 和正整数 $n>1$, 由文献 $[1,253$ 页, 辅理 $1.2]$, 可得 $\left[z^{n}, g\right]=[z, g]\left[z, g, z^{n-1}\right]\left[z^{n-1}, g\right]$. 又由 $K_{3}(G) \leqslant Z(G)$ 和 [1, 253 页, 辅理 1.3], 可以得到 $\left[z, g, z^{n-1}\right]=\left[z^{n-1},[z, g]\right]^{-1}=\left([z,[z, g]]^{n-1}\right)^{-1}=[z, g, z]^{n-1}$, 从而我们有 $\left[z^{n}, g\right]=$ $[z, g]\left[z^{n-1}, g\right][z, g, z]^{n-1}$. 因此, 用归纳法容易证明 $\left[z^{n}, g\right]=[z, g]^{n}[z, g, z]^{\frac{n(n-1)}{2}}$, 其中 $n>1$. 取 $n=p$, 并且由 $G^{\prime} \cong C_{p}^{3}$ 和 $p>2$, 我们得到 $\left[z^{p}, g\right]=[z, g]^{p}[z, g, z]^{\frac{p(p-1)}{2}}=1$. 这说明了 $\mho(G)=\left\langle z^{p} \mid z \in G\right\rangle \leqslant Z(G)$. 又因为 $\Phi(G)=G^{\prime} \mho(G)$, 所以 $[\Phi(G), g]=\langle[a, b, g]\rangle$. 于是对任 意的 $x \in \Phi(G)$ 和 $g, h \in G,[x, g]^{h}=\left([a, b, g]^{i}\right)^{h}=[a, b, g]^{i}$. 令 $M$ 是 $G$ 的一个极大子群. 因 为 $d(G)=2$, 所以 $M / \Phi(G)=\langle\bar{g}\rangle$, 其中 $g \in G$, 于是 $M=\Phi(G)\langle g\rangle$. 又由文献 [1,258 页, 辅理 $1.11]$ 得 $M^{\prime}=\left\langle[x, g]^{h} \mid x \in \Phi(G), h \in M\right\rangle=\langle[a, b, g]\rangle$, 从而 $\left|M^{\prime}\right| \leqslant p$. 因此 $G$ 是一个 $D_{1}$-群.

(iv) 因为 $c(G)=2$ 且 $G^{\prime} \cong C_{p}^{3}$ 或 $C_{p}^{2}$, 所以 $G^{\prime} \leqslant Z(G)$ 且 $\exp \left(G^{\prime}\right)=p$. 从而对任意 的 $x, y \in G$, 我们有 $\left[x^{p}, y\right]=[x, y]^{p}=1$, 故 $\mho(G) \leqslant Z(G)$. 于是 $\Phi(G)=G^{\prime} \mho(G) \leqslant Z(G)$. 因为 $d(G)=3$, 所以 $G / \Phi(G)$ 是一个 $p^{3}$ 阶初等交换 $p$-群, 并且 $G$ 的任意极大子群可以写 为 $M=\left\langle\Phi(G), g_{1}, g_{2}\right\rangle=\Phi(G)\left\langle g_{1}, g_{2}\right\rangle$. 由文献 [1,258 页, 辅理 1.11] 可得 $M^{\prime}=\left\langle[x, y]^{h}\right| x, y \in$ $\left.\Phi(G) \cup\left\{g_{1}, g_{2}\right\}, h \in M\right\rangle=\left\langle\left[g_{1}, g_{2}\right]\right\rangle$, 从而 $\left|M^{\prime}\right| \leqslant p$. 因此 $G$ 是一个 $D_{1}$-群.

\section{3 主要定理及其推论}

定理 3.1 设 $G$ 是一个有限 $p$-群, 那么 $G$ 为 $D_{1}$-群当且仅当下列结果之一成立:

(i) $\left|G^{\prime}\right| \leqslant p$;

(ii) $d(G)=2,\left|G^{\prime}\right|=p^{2}$;

(iii) $d(G)=2, c(G)=3, G^{\prime} \cong C_{p}^{3}$, 其中 $p>2$;

(iv) $d(G)=3, c(G)=2, G^{\prime} \cong C_{p}^{3}$ 或者 $C_{p}^{2}$.

证明 若 $G$ 为定理 3.1 的情形 (i)-(iv) 之一, 由引理 $2.9, G$ 是一个 $D_{1}$-群. 反之, 假设 $G$ 是一个 $D_{1}$-群. 只需证明 $G$ 为定理 3.1 的情形 (i)-(iv) 之一即可. 首先, 对 $G$ 的任意极大 子群 $M$, 由引理 2.6 , 我们有 $\left|M^{\prime}\right|=1$ 或 $p, M^{\prime} \leqslant G^{\prime} \cap Z(G)$. 由引理 $2.7,\left|G^{\prime}\right| \leqslant p^{3}$, 并且当 $\left|G^{\prime}\right| \geqslant p^{2}$ 时 $d(G) \leqslant 3$, 所以我们分下列三种情况来完成证明. 
情况 $1 \quad\left|G^{\prime}\right| \leqslant p$.

$G$ 为定理 3.1 的情形 (i).

情况 $2\left|G^{\prime}\right|=p^{2}$ 且 $d(G) \leqslant 3$.

由定理 $1.1, G$ 既非交换也非内交换. 因此存在一个 $G$ 的极大子群 $A$ 满足 $\left|A^{\prime}\right|=p$. 如果 对 $G$ 的任意极大子群 $M, M^{\prime} \leqslant A^{\prime}$, 那么 $G / A^{\prime}$ 的任意极大子群交换. 再由 $\left|G^{\prime} / A^{\prime}\right|=p>1$, 得 $G / A^{\prime}$ 非交换, 所以 $G / A^{\prime}$ 为内交换 $p$-群. 由 $A^{\prime} \leqslant \Phi(G)$ 和定理 1.1, 得 $d(G)=d\left(G / A^{\prime}\right)=2$. $G$ 为定理 3.1 的情形 (ii). 如果 $G$ 有极大子群 $B$ 满足 $B^{\prime} \nless A^{\prime}$. 由于 $A^{\prime} \leqslant Z(G), B^{\prime} \leqslant Z(G)$ 且 $\left|G^{\prime}\right|=p^{2}$, 我们有 $G^{\prime}=A^{\prime} B^{\prime} \leqslant Z(G)$ 且 $G^{\prime} \cong C_{p}^{2}$. 若 $G=\langle a, b\rangle$, 则由 $G^{\prime} \leqslant Z(G)$, 得 $G^{\prime}=\langle[a, b]\rangle$, 矛盾于 $G^{\prime} \cong C_{p}^{2}$. 若 $d(G)=3$, 由 $G^{\prime} \leqslant Z(G)$, 我们得到 $c(G)=2, G$ 为定理 3.1 的情形 (iv).

情况 $3\left|G^{\prime}\right|=p^{3}$ 且 $d(G) \leqslant 3$.

由引理 2.4, 存在 $G$ 的极大子群 $A$ 和 $B$ 满足 $\left|A^{\prime} B^{\prime}\right|=p^{2}$. 又由引理 $2.6, A^{\prime} B^{\prime} \leqslant Z(G)$ 且 $A^{\prime} B^{\prime}=A^{\prime} \times B^{\prime} \cong C_{p}^{2}$. 因为 $G^{\prime} / A^{\prime} B^{\prime}$ 循环且 $A^{\prime} B^{\prime} \leqslant Z(G)$, 所以 $G^{\prime}$ 交换, $G$ 亚交换. 从而 $G^{\prime}=C_{p^{2}} \times C_{p}$ 或 $G^{\prime} \cong C_{p}^{3}$. 因为 $G^{\prime} \neq A^{\prime} B^{\prime}$, 所以 $G / A^{\prime} B^{\prime}$ 是一个非交换 $p$-群.

假设对 $G$ 的任意极大子群 $M$ 有 $M^{\prime} \leqslant A^{\prime} B^{\prime}$. 那么 $G / A^{\prime} B^{\prime}$ 的任意极大子群交换, 所以 $G / A^{\prime} B^{\prime}$ 是一个内交换 $p$-群. 如果 $G / A^{\prime} B^{\prime}$ 亚循环, 由引理 2.3 得 $G$ 亚循环, 则 $G^{\prime}$ 循环, 这 矛盾于 $A^{\prime} B^{\prime} \cong C_{p}^{2}$. 因此 $G / A^{\prime} B^{\prime}$ 非亚循环. 由定理 1.1 , 我们可以假设

$$
G / A^{\prime} B^{\prime}=\bar{G}=\left\langle\bar{a}, \bar{b} \mid \bar{a}^{p^{n}}=\bar{b}^{p^{m}}=\bar{c}^{p}=\overline{1},[\bar{a}, \bar{b}]=\bar{c},[\bar{c}, \bar{a}]=\overline{1},[\bar{c}, \bar{b}]=\overline{1}\right\rangle,
$$

其中如果 $p=2$, 那么 $m+n \geqslant 3$. 我们容易得到 $K_{2}(\bar{G})=\langle\bar{c}\rangle$ 且 $K_{3}(\bar{G})=1$. 因为 $A^{\prime} B^{\prime} \leqslant \Phi(G) \cap Z(G)$, 我们有 $K_{3}(G) \leqslant Z(G)$, 即 $K_{4}(G)=1$. 令 $a$ 和 $b$ 分别是 $\bar{a}$ 和 $\bar{b}$ 在 $G$ 中的原像, 并且令 $[a, b]=d$. 那么 $G=\langle a, b, \Phi(G)\rangle=\langle a, b\rangle$, 并且 $\bar{d}=\bar{c}$. 设 $[d, a]=z_{1}$, $[d, b]=z_{2}$, 其中 $z_{1}, z_{2} \in A^{\prime} B^{\prime}$. 由文献 $\left[1,258\right.$ 页, 1.11] 得 $G^{\prime}=\left\langle[a, b], K_{3}(G)\right\rangle$. 因为 $[b, a, b]=z_{2}^{-1},[b, a, a]=z_{1}^{-1}$ 且 $K_{4}(G)=1$, 由引理 2.9 (iii) 的证明, 我们得到 $K_{3}(G)=$ $\langle[a, b, b],[a, b, a],[b, a, b],[b, a, a]\rangle=\left\langle z_{1}, z_{2}\right\rangle$. 于是 $G^{\prime}=\left\langle d, z_{1}, z_{2}\right\rangle$. 如果 $z_{i} \in\langle d\rangle, i=1,2$, 那 么 $G^{\prime}=\langle d\rangle$, 矛盾于 $A^{\prime} \times B^{\prime} \leqslant G^{\prime}$. 因此 $z_{1}$ 或者 $z_{2} \notin\langle d\rangle$. 不失一般性, 令 $z_{2} \notin\langle d\rangle$. 因为 $K_{3}(G) \leqslant Z(G)$, 用引理 2.9 (iii) 的证明方法, 我们得到 $\left[a^{p}, b\right]=d^{p} z_{1}^{\frac{p(p-1)}{2}}$ 且 $\left[a, b^{p}\right]=d^{p} z_{2}^{\frac{p(p-1)}{2}}$.

假设 $G^{\prime} \cong C_{p^{2}} \times C_{p}$, 那么 $A^{\prime} B^{\prime}=\left\langle d^{p}, z_{2}\right\rangle$. 因为 $z_{i} \in A^{\prime} \times B^{\prime}$, 我们有 $|d|=p^{2}$. 由于 $\Phi(G)=G^{\prime} \mho(G)$ 且 $d(G)=2$, 于是对任意的 $x \in G,\left\langle x, G^{\prime} \mho(G)\right\rangle$ 是 $G$ 的真子群. 若 $p>2$, 则 $z_{1}^{\frac{p(p-1)}{2}}=1$ 且 $\left[a^{p}, b\right]=d^{p}$. 因为 $a^{p}$ 和 $d$ 都在 $G^{\prime} \mho(G)$ 中, 我们有 $\left\langle b, G^{\prime} \mho(G)\right\rangle^{\prime} \geqslant\left\langle d^{p}, z_{2}\right\rangle \cong C_{p}^{2}$, 矛盾. 若 $p=2$, 由 $z_{1} \in A^{\prime} B^{\prime}=\left\langle d^{2}, z_{2}\right\rangle$, 我们得到 $z_{1}=1, d^{2}, z_{2}$ 或 $d^{2} z_{2}$. 若 $z_{1}=1$, 则 $\left[a^{2}, b\right]=d^{2}$, 所以 $\left\langle b, G^{\prime} \mho(G)\right\rangle^{\prime} \geqslant\left\langle d^{2}, z_{2}\right\rangle \cong C_{2}^{2}$. 若 $z_{1}=d^{2}$ 或 $z_{2}$, 则 $\left[a, b^{2}\right]=d^{2} z_{2}$. 由 $[d, a]=d^{2}$ 或 $z_{2}$, 我们有 $\left\langle a, G^{\prime} \mho(G)\right\rangle^{\prime} \geqslant\left\langle d^{2}, z_{2}\right\rangle \cong C_{2}^{2}$. 若 $z_{1}=d^{2} z_{2}$, 则 $\left[a^{2}, a b\right]=\left[a^{2}, b\right]=d^{2} z_{1}=z_{2}$ 且 $[d, a b]=[d, a][d, b]=z_{1} z_{2}=d^{2}$, 所以 $\left\langle a b, G^{\prime} \mho(G)\right\rangle^{\prime} \geqslant\left\langle d^{2}, z_{2}\right\rangle \cong C_{2}^{2}$. 综上所述, 我们得到 $G$ 有 一个真子群 $M$ 满足 $M^{\prime} \geqslant\left\langle d^{2}, z_{2}\right\rangle \cong C_{2}^{2}$, 这矛盾于 $G$ 是一个 $D_{1}$-群.

如果 $G^{\prime} \cong C_{p}^{3}$, 那么 $d^{p}=1$ 且 $G^{\prime}=\langle d\rangle \times\left\langle z_{1}\right\rangle \times\left\langle z_{2}\right\rangle$. 若 $p=2$, 因为 $\exp \left(G^{\prime}\right)=p$, 我们 有 $\left[a, b^{2}\right]=[a, b, b]=z_{2}$, 然而 $G$ 有极大子群 $\left\langle a^{2}, b^{2}, d, z_{1}, z_{2}, a\right\rangle$, 它的导群为 $\left\langle z_{1}, z_{2}\right\rangle$, 矛盾于 $G$ 是一个 $D_{1}$-群. 若 $p>2, G$ 为定理 3.1 的情形 (iii).

假设 $G$ 有三个极大子群 $A, B$ 和 $C$ 满足 $\left|A^{\prime} B^{\prime} C^{\prime}\right|=p^{3}$, 那么 $G^{\prime}=A^{\prime} B^{\prime} C^{\prime}=A^{\prime} \times B^{\prime} \times C^{\prime} \leqslant$ 
$Z(G)$, 从而 $G^{\prime} \cong C_{p}^{3}$. 若 $d(G)=2$, 令 $G=\langle a, b\rangle$. 因为 $G^{\prime} \leqslant Z(G)$, 由文献 [1, 258 页, 1.11], 我们有 $G^{\prime}=\langle[a, b]\rangle$, 矛盾于 $G^{\prime} \cong C_{p}^{3}$. 所以 $d(G) \neq 2$. 若 $d(G)=3, G$ 为定理 3.1 的情形 (iv). 证明完成.

由定理 3.1, 我们很容易给出 $D_{1}$-群的另一描述.

推论 3.2 设 $G$ 是一个有限 $p$-群, 那么 $G$ 是一个非交换 $D_{1}$-群当且仅当下列结果之一 成立

(i) $G=\left(A_{1} * A_{2} * \cdots * A_{s}\right) Z(G)$, 其中 $*$ 表示中心积, $A_{1}, \ldots, A_{s}$ 为 $G$ 的内交换子群;

(ii) $G$ 亚循环且 $\left|G^{\prime}\right|=p^{2}$;

(iii) $G^{\prime} \cap Z(G)$ 中存在子群 $N$ 使得 $|N|=p$ 或 $N \cong C_{p}^{2}, G / N$ 为非亚循环内交换 $p$-群;

(iv) $G^{\prime} \cap Z(G)$ 中存在子群 $N$ 使得 $N \cong C_{p}^{3}$ 或 $C_{p}^{2}, G / N$ 交换.

证明 我们按定理 3.1 的 (i)-(iv) 四种情形来完成证明. 在情形 (i) 中, 我们有 $\left|G^{\prime}\right|=p$, 于是结论成立 (参见定理 1.2). 在情况 (ii) 中, $G^{\prime} \cap Z(G)$ 中存在子群 $N$, 使得 $|N|=p$, $d(G / N)=2$ 且 $\left|(G / N)^{\prime}\right|=p$. 由引理 2.8 容易得到 $G / N$ 是一个内交换 $p$-群. 再由定理 3.1 的证明, 有两种可能

(1) $G$ 亚循环且 $\left|G^{\prime}\right|=p^{2}$, 或者

(2) $G^{\prime} \cap Z(G)$ 中存在 $p$ 阶子群 $N$ 使得 $G / N$ 为非亚循环内交换 $p$-群.

在情况 (iii) 中, 由定理 3.1 的证明, $G^{\prime} \cap Z(G)$ 中存在子群 $N$ 使得 $N \cong C_{p}^{2}, G / N$ 为 非亚循环内交换 $p$-群. 在情况 (iv) 中, 因为 $c(G)=2$, 所以 $G^{\prime} \cap Z(G)$ 中存在子群 $N$ 使得 $N \cong C_{p}^{3}$ 或 $C_{p}^{2}, G / N$ 交换. 结论成立.

致谢感谢 Berkovich 教授和审稿人, 他们仔细阅读了本文的初稿并且提供了一些有用的 建议和帮助.

\section{参考文献}

1 Huppert B. Endliche Gruppen I. New York-Berlin: Springer, 1967

2 Rédei L. Das schiefe product in der grouppentheorie. Comment Math Helvet, 20: 225-267 (1947)

3 Berkovich Y. On subgroups of finite p-groups. J Algebra, 224: 198-240 (2000)

4 Blackburn S R. Groups of prime power order with derived subgroup of prime order. J Algebra, 219: 625-657 (1999)

5 Berkovich Y. Group of Prime Power Order, Book I, in preparation

6 Berkovich Y. Finite $p$-groups with few minimal non-abelian subgroups. J Algebra, 297: 62-100 (2006)

7 Tuan H F. A theorem on metabelian p-groups and some consequences. Chin Ann Math Ser B, 5B: 1-6 (1984)

8 Isaacs I M. Character Theory of Finite Groups. New York: Academic Press, 1976

9 Berkovich Y. Short proofs of some basic characterization theorems of finite p-group theory. Glas Math, 41: 239-258 (2006)

10 Berkovich Y, Janko Z. Structure of finite p-group with given subgroups. Contemp Math, 402: 13-93 (2006) 